\title{
Central Nervous System Changes in Pediatric Heart Failure: A Volumetric Study
}

\author{
Jondavid Menteer $\cdot$ Paul M. Macey $\cdot$ \\ Mary A. Woo $\cdot$ Ashok Panigrahy $\cdot$ Ronald M. Harper
}

Received: 5 February 2010/Accepted: 5 May 2010/Published online: 3 June 2010

(C) The Author(s) 2010. This article is published with open access at Springerlink.com

\begin{abstract}
Autonomic dysfunction, mood disturbances, and memory deficits appear in pediatric and adult heart failure (HF). Brain areas controlling these functions show injury in adult HF patients, many of whom have comorbid cerebrovascular disease. We examined whether similar brain pathology develops in pediatric subjects without such comorbidities. In this study, high-resolution $\mathrm{T} 1$ brain magnetic resonance images were collected from seven severe HF subjects age (age 8-18 years [mean 13]; left ventricular shortening 9 to $19 \%$ [median 14\%]) and seven age-matched healthy controls (age 8-18 years [mean 13]). After segmentation into gray matter (GM), white matter, and cerebrospinal fluid (CSF), regional volume loss between groups was determined by voxel-based morphometry. GM volume loss appeared on all HF scans, but ischemic changes and infarcts were absent. HF subjects
\end{abstract}

J. Menteer ( $\square)$

Division of Cardiology, Childrens Hospital

Los Angeles, Los Angeles, CA 90027, USA

e-mail: jmenteer@chla.usc.edu

P. M. Macey - M. A. Woo

School of Nursing, University of California at Los Angeles, Los Angeles, CA 90095, USA

P. M. Macey · R. M. Harper

Brain Research Institute, University of California at Los

Angeles, Los Angeles, CA 90095, USA

A. Panigrahy

Department of Radiology, Childrens Hospital Los Angeles,

Los Angeles, CA 90027, USA

R. M. Harper

Department of Neurobiology, David Geffen School of Medicine, University of California at Los Angeles, Los Angeles,

CA 90095, USA showed greater CSF volume than controls (mean $\pm \mathrm{SD}$ $0.30 \pm 0.04$ vs. $0.25 \pm 0.041, P=0.03)$, but total intracranial volume was identical $(1.39 \pm 0.11$ vs. $1.39 \pm$ $0.091, P=$ NS). Regional GM volume reduction appeared in the right and left posterior hippocampus, bilateral midinsulae, and the superior medial frontal gyrus and midcingulate cortex of HF subjects (threshold $P<0.001$ ). No volume-loss sites appeared in control brains. We conclude that pediatric HF patients show brain GM loss in areas similar to those of adult HF subjects. Substantial changes emerged in sites that regulate autonomic function as well as mood, personality and short-term memory. In the absence of thromboembolic disease and many comorbid conditions found in adult HF patients, pediatric HF patients show significant, focal GM volume loss, which may coincide with the multiple neurologic and psychological changes observed in patients with HF.

Keywords Central nervous system - Heart failure · Magnetic resonance imaging $\cdot$ Pediatrics

\section{Introduction}

Chronic heart failure (HF) has been associated with many comorbid symptoms and physiologic abnormalities, including cognitive impairment [2, 44, 45], depression [23, $34,51]$, anxiety [26, 34, 47], impulsivity [28, 54], and altered autonomic regulation $[15,32,35]$ in both adults and children.

In adult patients, chronic HF is accompanied by severe injury to the central nervous system (CNS) structures that regulate the cardiovascular system, including the hypothalamus, insular cortex, cingulate gyrus, the basal forebrain, cerebellar cortex and deep nuclei, and portions of the 
hippocampus [55]. The injury appears as loss of tissue in specific brain areas [55], which is confirmed by impaired functional neural responses to cardiovascular challenges mediated by those brain structures [56]. Brain injury may therefore contribute to multiple morbidities in patients with $\mathrm{HF}$ syndrome and may exacerbate progression of the condition [41].

However, age-related CNS changes, including infarctions and brain volume shrinkage, occur in many adults with [3, 46] and without [19] HF, potentially confounding the anatomic findings. Pediatric subjects do not commonly show such CNS degenerative changes and often have fewer comorbid conditions, such as diabetes and smoking that can induce brain damage.

We sought to determine whether CNS changes found in adult HF subjects appear in the pediatric condition, thus helping to explain some of the comorbid pathologies, such as the dysautonomia, including exaggerated sympathetic outflow $[6,36]$, that likely contribute to exacerbation of the condition. Given the common occurrence of dysautonomic symptoms [32, 33, 35], dyspnea [33], school performance issues [12, 30, 31, 49, 53], and mood disorders [47] in pediatric HF patients, we hypothesized that brain injury would appear in areas important for control of sympathetic and parasympathetic outflow (e.g., insular and anterior cingulate cortices), memory (e.g., the hippocampus and/or mammillary bodies), and/or mood regulation (e.g., hippocampus, hypothalamus).

\section{Methods}

\section{Study Design}

The study design was a case-control cohort study of pediatric patients, age 7 to 18 , versus age-matched healthy control subjects, in a single, large pediatric center. We collected high-resolution three-dimensional (3D) magnetic resonance imaging (MRI) brain scans from pediatric subjects with chronic HF and sought focal differences in gray matter (GM) volume within the brain, relative to agematched healthy control subjects, while partitioning the effects of sex and brain size. The studies were approved by the performing hospital's Committee for Clinical Investigations, and subjects, as well as parents and guardians, gave written informed consent. The recruitment period was 13 months.

\section{Study Population}

We recruited HF patients with primary cardiomyopathy, age 7-18, with left ventricular (LV) shortening fractions $<21 \%$ on a chronic basis who had no past history of neurologic injury or neuropsychiatric disease. HF therapies, including use of inotropes, beta-blockers, or diuretics, were not controlled. Patients with contraindications for MRI and patients with CNS disease or abnormality were excluded. Patients $<7$ years of age were not included because such patients typically are unable to cooperate with awake MRI examination.

A matched control population, with images obtained using identical techniques and resolution, is critical to the validity and sensitivity of the statistical methods described later. Because our research MRI scan procedure differed from our institution's clinical scan procedure, we were unable to use clinical scans as a control population. We therefore recruited control subjects who were either siblings of affected subjects or unrelated healthy children recruited from the cardiology clinics (after evaluation that showed no cardiac disease).

Because anatomic abnormalities can introduce errors into statistical analysis, two subjects (1 with HF and 1 control) were excluded after MRI scanning: one HF subject due to an incidental MRI finding of a tiny cavernous hemangioma and one control subject after an incidental MRI finding of a small arachnoid cyst. Another 7 year-old control subject was recruited but later excluded because of inability to cooperate with the MRI evaluation.

\section{MRI}

Patients underwent nonsedated brain MRI using a General Electric Signa 1.5-Tesla MRI scanner (General Electric Healthcare, CT, USA) with a standard adult quadrature head coil. After scout imaging, a minimum of three volumes of T1 3D fast spoiled gradient-recalled-echo data were acquired (1.2-mm slice thickness, $256 \times 256$ matrix, $26-\mathrm{cm}$ field of view, $22^{\circ}$ flip angle, no interslice gap, 10,100-ms repetition time, 2-ms echo time, one excitation). Any sequence with visible movement artifact was repeated. This sequence requires more scan time but produces much higher quality images than the standard stacked T1 images obtained per routine for our institution's clinical scans.

\section{Postprocessing}

Data were uploaded in DICOM format to a networked laboratory computer for analysis and converted to the NIfTI format [11] for analysis in SPM5. We used Statistical Parametric Mapping (SPM5, University College London) software to perform the image analysis.

Voxel-based morphometry (VBM) [5] was used to identify areas of the brain that had lower or higher likelihood of being GM in HF versus control subjects. This procedure included several steps. First, each subject's repeated brain volumes were realigned and averaged to 
improve the signal-to-noise ratio. Second, the SPM5 unified segmentation procedure was applied to the averaged image to correct for intensity variations from field inhomogeneities, segment GM, white matter (WM), and cerebrospinal fluid (CSF) (Fig. 1) and to calculate spatial normalization parameters [5]. These parameters were used to transform the GM images into a common space. Next, the total volume of each tissue segment was computed, in liters, from the segmented images by calculating the volume of all voxels with $P>0.5$ of belonging to the tissue type (i.e., GM, WM, or CSF). Finally, the normalized GM segments were smoothed with a Gaussian filter having a kernel size of $12 \mathrm{~mm}$ (full-width-at-half-maximum) [5]. Statistics were performed on the realigned, averaged, segmented, smoothed volumes.

\section{Statistics}

Statistics were performed on the whole brain after segmentation as well as on a voxel-by-voxel basis. After segmentation (Fig. 1), whole-brain tissue volume quantization was performed (Table 1). Mean CSF volume, GM volume,
$\mathrm{WM}$ volume, total brain volume $(\mathrm{TBV}=\mathrm{GM}+\mathrm{WM})$, and total intracranial volume $(\mathrm{TIV}=\mathrm{GM}+\mathrm{WM}+\mathrm{CSF})$ were calculated (Table 1). Student $t$ tests were used to study the differences in whole-brain volumes.

Regional differences between HF and control groups were determined using analysis of covariance at all locations in the brain with segmented GM $P>0.5$, and age, total intracranial volume, and sex were included as covariates. We used a statistical threshold of $P<0.001$. The regions of significant volume reduction were superimposed on the mean of all subjects' normalized T1 images using MRIcroN software (Chris Rorden, http://www. cabiatl.com/mricro/mricron). The images were saved as color NIfTI files and visualized in 3D using MRIcroGL (Chris Rorden, http://www.cabiatl.com/mricro/mricrogl).

\section{Results}

Seven HF subjects (age 7-18 [mean 12.9] with LV shortening fraction $14 \pm 3 \%$ ) and seven control subjects (age 8-17 years [mean 12.9] and age-matched within 1 year of

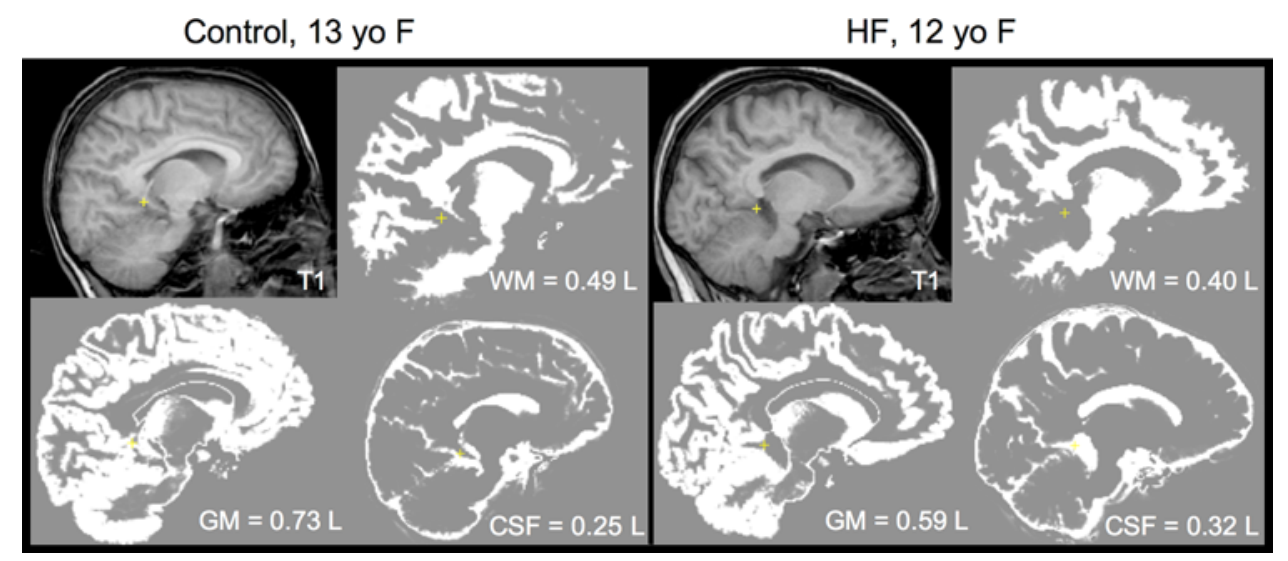

Fig. 1 Demonstration of segmentation of brain tissue. Separation of GM from WM and CSF is necessary to demonstrate volume changes by VBM. These panels exemplify the concept of segmentation using images from the scans of one study subject and one control as examples. Using a standardized probability map, high-resolution 3D T1 scans were segmented into GM, WM, and CSF voxels, and the total volume depicted by the voxels in each volume was calculated.
The left panel depicts a slice of the intact and segmented T1 scan of a control subject. The right panel depicts a slice of the intact and segmented scan of an age-matched patient with HF. Although the differences are difficult to appreciate visually, this separation allows the computation of the volumes of CSF, GM, and WM for each subject. The total calculated volume of each compartment, in liters, is displayed on each image
Table 1 Whole-brain statistics as derived from tissuesegmented MRI images

Values represent average volumes of the respective tissues in liters

\begin{tabular}{|c|c|c|c|c|c|}
\hline \multirow[t]{2}{*}{ Statistics } & \multicolumn{2}{|l|}{$\mathrm{HF}(n=7)$} & \multicolumn{2}{|c|}{ Controls $(n=7)$} & \multirow[b]{2}{*}{$P$} \\
\hline & Volume & $\%$ TIV & Volume & $\%$ TIV & \\
\hline Gray matter & $0.65 \pm 0.07$ & $47 \pm 3$ & $0.69 \pm 0.04$ & $49 \pm 2$ & NS \\
\hline WM & $0.44 \pm 0.04$ & $31 \pm 2$ & $0.45 \pm 0.03$ & $32 \pm 2$ & NS \\
\hline Cerebrospinal fluid & $0.30 \pm 0.04$ & $22 \pm 2$ & $0.25 \pm 0.04$ & $18 \pm 2$ & 0.03 \\
\hline TIV & $1.39 \pm 0.11$ & 100 & $1.39 \pm 0.09$ & 100 & NS \\
\hline Brain volume $(\mathrm{GM}+\mathrm{WM})$ & $1.09 \pm 0.09$ & $78 \pm 2$ & $1.14 \pm 0.06$ & $82 \pm 2$ & NS \\
\hline Gray-to-white ratio & $1.50 \pm 0.17$ & & $1.52 \pm 0.09$ & & NS \\
\hline
\end{tabular}


HF subjects) were included in the analysis. New York Heart Association classes of HF subjects were II $(n=2)$, class III $(n=3)$, and class IV $(n=2)$. All HF subjects were receiving loop diuretics. None were receiving anticonvulsants or steroids.

\section{MRI Findings}

A single neuroradiologist, blinded to the subjects' cardiac status, assessed all MRI scans for evidence of clinical abnormalities. All seven HF scans showed mild global volume loss. No HF subject had evidence of an old infarct, and no calcification was noted. None of the controls showed abnormal findings.

\section{Whole-Brain Analysis}

From whole-brain statistics, CSF volume was significantly increased in $\mathrm{HF}$ subjects $(0.30 \mathrm{l} / \mathrm{HF}$ subject vs. $0.25 \mathrm{l} /$ control, $P=0.03)$. There was a trend toward global GM loss $\quad(0.65 \mathrm{l} / \mathrm{HF}$ subject vs. $0.691 /$ control, $P=$ not significant [NS]) and decreased TBV (1.09 1 for HF vs. 1.141 for controls, $P=\mathrm{NS})$. Average TIV was identical between groups (1.39 1), and no significant differences appeared between WM volume (0.44 1 for HF vs. 0.451 for controls) or GM-to-WM ratio (1.50 for HF vs. 1.52 for controls).

\section{Regional Analysis}

Several brain regions showed decreased GM volume in HF subjects compared with controls. We detected no foci of greater GM volume in HF subjects (i.e., no GM loss was detected in controls).

The affected areas included both limbic and cortical regions. The mid-to-posterior insula showed GM loss bilaterally (Fig. 2). The hippocampus was affected bilaterally in the posterior region, with greater injury on the right side extending to the right mid-hippocampus (Fig. 3). The medial aspect of the superior frontal gyrus and the adjacent mid-cingulate cortex were affected (Fig. 4). Cortical areas of decreased volume included areas in the
Fig. 2 Insular injury. Areas of GM volume loss $(P<0.001)$ in the bilateral insular cortices $(a$ through $d$ ) in pediatric HF. In the left panel, the area of significant change is superimposed onto an image of the average brain of all normal subjects $(a$ and $b)$. In the right panel, for clarity, the same data are superimposed onto an image of a single control subject's brain $(c$ and $d)$
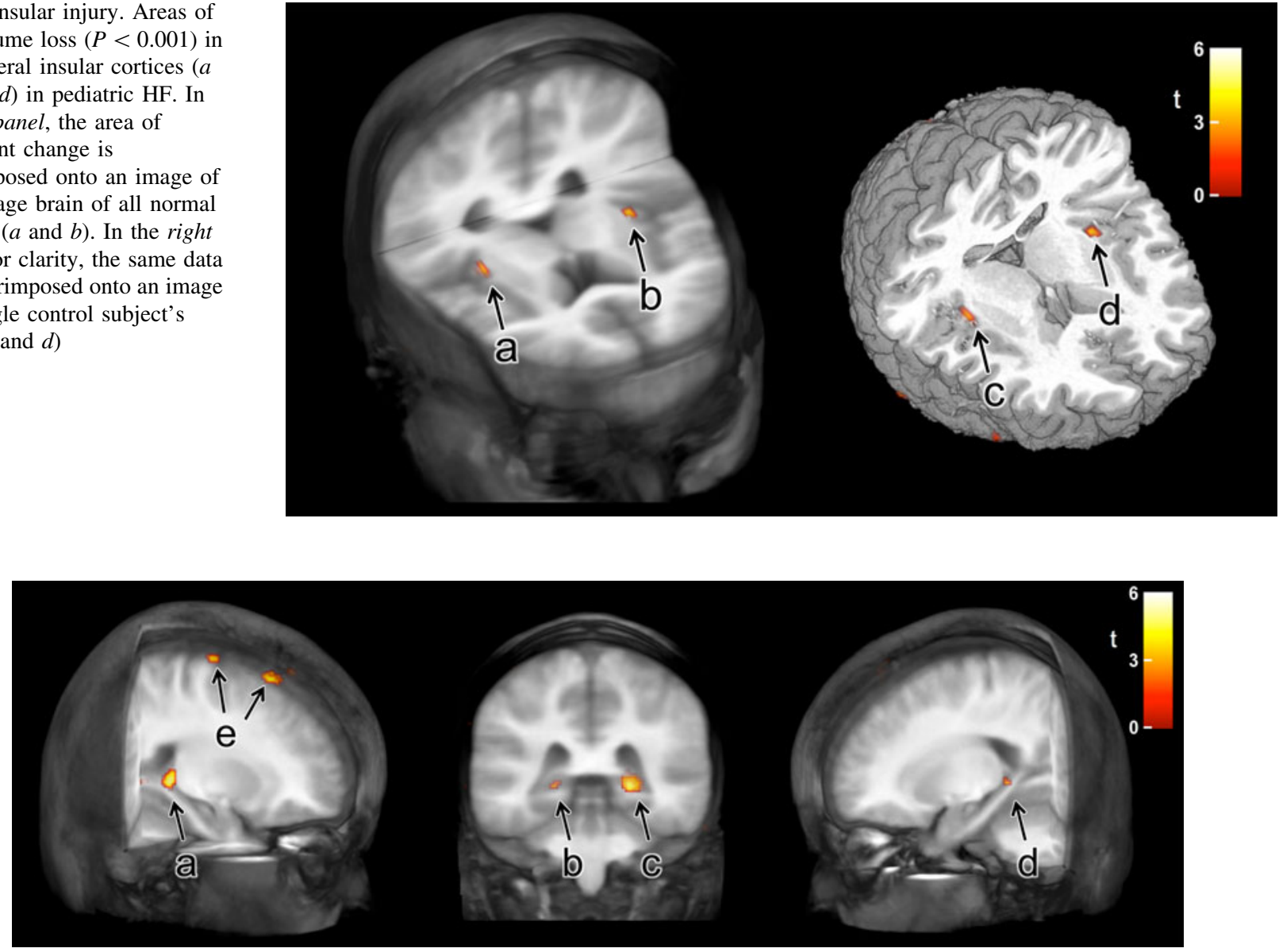

Fig. 3 Posterior hippocampus injury. Areas of GM volume loss $(P<0.001)$ in the right $(a$ and $c)$ and left $(b$ and $d)$ posterior hippocampus in pediatric HF subjects are superimposed onto three projections of the averaged brain of all normal subjects. Two small areas of volume loss $(e)$ also appear in the dorsal right frontal cortex 
Fig. 4 Superior frontal gyrus and mid-cingulate injury. Areas of GM volume loss $(P<0.001)$ in the medial aspect of the superior frontal gyrus extending into the anterior portion of the mid-cingulate $(a$ and $b$ ) in pediatric HF subjects are superimposed onto the average image of all normal subjects. The ventral extent of the right hippocampal region of interest (c) is also apparent as is a focus of parietal cortical volume loss $(d)$

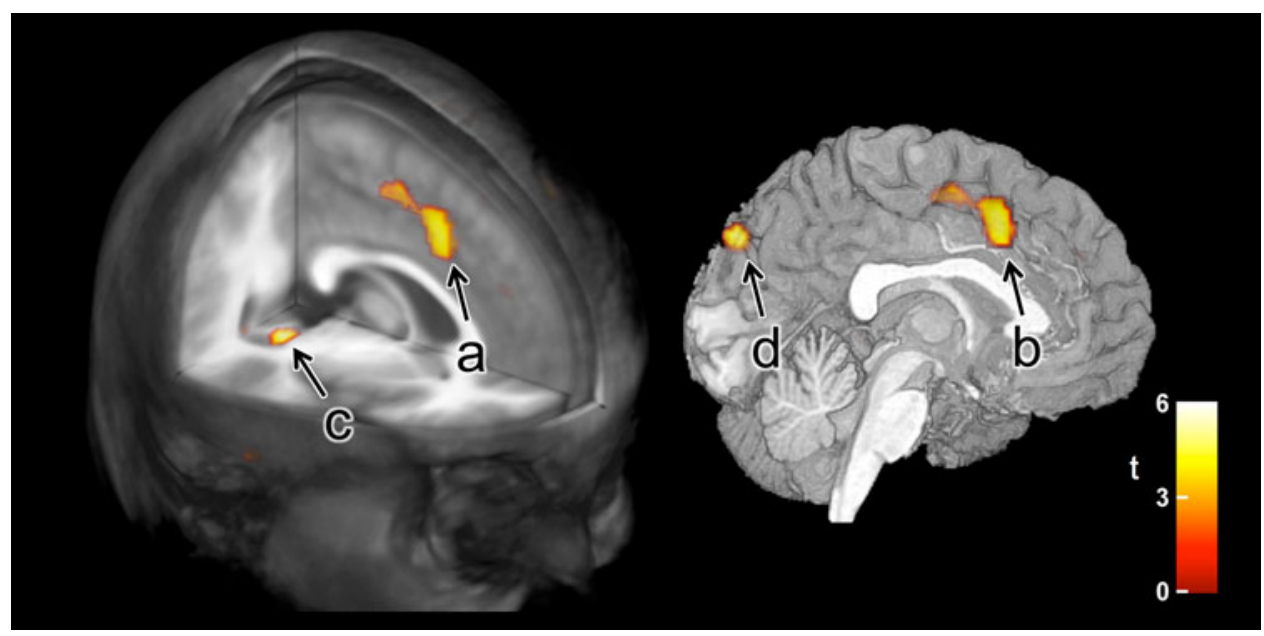

superior frontal cortex (Fig. 3), and parietal sites (primarily bilateral sensory regions [Fig. 4]). Other isolated areas of loss appeared in the lateral temporal cortices as well as the medial parietal-occipital sulcus.

\section{Discussion}

We demonstrated that in a group of pediatric chronic HF subjects, GM volume loss is common and occurs in specific areas of the brain, including the insulae, superior frontal gyrus, and posterior hippocampus, which are important for cardiovascular homeostasis, mood, and memory. The findings in our HF subjects appear to parallel the injury found in adult HF patients [55]. The nature and severity of changes in adult HF appear more extensive [55], which may stem from (1) the greater statistical power associated with the larger population in that study, (2) the longer illness duration in adults, or (3) the presence of comorbidities accompanying the older patient population [19].

Although volume loss is not synonymous with damage, previous work has demonstrated depressed or altered brain activity by functional MRI in similar instances of GM volume loss $[21,22,24,56]$. This information suggests that children may not be immune to the CNS injuries found in adult HF patients. Indeed, it is likely that the brain is affected by HF in ways unrelated to ischemia and age. Collectively, the findings suggest a neurologic basis for the deficits in autonomic regulation [32, 33], cognition [5, 46, 50], and mood [12, 47, 49] in pediatric HF patients.

In the present study, global brain volume loss appeared in every subject with chronic HF. The subjective volume loss corresponds to a significantly greater volume of CSF and a more modest trend toward reduction in WM and GM volumes. Global brain volume loss has been described in MRI studies of cyanotic heart disease in children and in adults with $\mathrm{HF}[18,52]$, and may correlate with poor neurologic outcomes, including learning disabilities, in young children [29, 31, 53]. The neurodevelopmental outcomes of children with isolated HF (not associated with anatomic heart abnormalities requiring surgery) has not been fully described.

We found localized loss of brain tissue in both the left and right insular cortices (Fig. 2). This finding is particularly important to the HF condition because the insular cortices provide a principal cortical influence to the hypothalamus and regulate both sympathetic and parasympathetic outflow [38-40]. Both adults and children with HF display a number of autonomic pathologies [33, 35], including enhanced sympathetic outflow $[6,36]$, which may be a precursor to clinical deterioration [20]. Injury to the insular cortex (especially the posterior aspect) consistently appears in studies of adult HF subjects [55]. HF subjects show inappropriate (decrease and phase-lagged) functional MRI responses to transient blood pressure challenges within the insular cortex [56]. Severity of autonomic dysregulation accompanies disease progression in HF in adults [20, 42] as well as children [32].

Insular damage may underlie and/or promote disease progression in HF. The insular cortices contribute to baroreflex sensitivity [58] and respond significantly to blood pressure challenges [22]. Insular tissue loss may contribute to the dyspnea common to pediatric and adult HF patients because the structure routinely activates in breathing challenges eliciting that perception [7, 43]. It also seems likely that injury to these regions contributes to arrhythmias and sudden death, events that stem from dysautonomia [37-40].

We also detected substantial volume loss in the inferomedial aspect of the superior frontal gyrus and the adjacent area of the mid-cingulate gyrus (Fig. 4). Damage to this area of the frontal lobe has been linked to effects on personality (disinhibition, irritability, apathy), changes in mood (mania, depression), obsessive-compulsive behaviors [10], and 
deficits in working memory[14]. Mid-anterior cingulate volume loss also appeared in earlier adult HF studies [55]. A relation between this structure, autonomic control, and, especially, blood pressure regulation has been described previously [8, 17, 21, 24]; the mid-cingulate has extensive projections to the insula and other limbic sites [4].

The posterior hippocampus also displayed volume loss, more pronounced on the right side (Fig. 3). The posterior hippocampus sends fibers through the fornix to the mammillary bodies. Injury to any component of this hippocampo-fornix-mammillary body circuit is accompanied by anterograde memory deficits $[1,16,27,28]$ and mood disorders, depression in particular $[9,13]$. Although memory deficits have not specifically been assessed in cases of severe pediatric HF, studies of psychological functioning soon after heart transplant suggest that this population remain at risk for depression and poor school performance [12, 49, 57]. Patients with other forms of severe cardiac disease in childhood are also at risk for depression, anxiety, and poor school performance [29, 31, 53]. The link between posterior hippocampal injury and these long-term neuropsychiatric issues requires further study.

Other areas with GM volume loss included isolated small areas within the motor cortex, the upper-to-midbody sensory cortex, and portions of the dorsal frontal cortex bilaterally. Loss of brain tissue and T2 MRI signals indicative of injury are consistently found in adults with $\mathrm{HF}$ and suggest common injurious processes in adult and pediatric HF pathophysiology.

In the absence of thromboembolic explanations, and given that the pattern of volume loss found here does not cleanly fit a "watershed" ischemia pattern of neuronal injury, the etiology of such brain changes remains poorly understood. Patients with severe HF may undergo decreased cerebral blood flow beyond the compensatory capacity of autoregulatory mechanisms, and injury may result from hypoxic processes, excitotoxicity, or programmed cell death, which may be accentuated in certain brain areas by atypical metabolic stress or vulnerability. Such atypical stress may be induced by a number of factors within HF syndrome, including chronic sympathetic overactivation [48], inflammation [25], or other hormonal abnormalities. It may be possible to partition the injurious processes by selective blockade of sympathetic processes, by regional assessment of perfusion, or by minimizing hypoxic exposure from sleep-related breathing pathology in the syndrome, but those studies remain to be performed.

We are not aware of any previous pediatric studies linking unoperated heart disease with specific CNS injury. This study demonstrates that regional brain volume loss occurs in pediatric HF patients and that the volume loss occurs in the absence of the focal ischemic changes that are common in both adult patients [46] and in postoperative children with congenital heart disease $[30,52]$. We therefore must consider that chronic $\mathrm{HF}$ exerts effects on important CNS functions independent of thromboembolic events or other vascular disease.

\section{Limitations}

Our small sample size generated only enough power to detect major differences/injuries between subject groups. The ability to discern focal volume loss in the present study, even with very low power, likely speaks to a large effect size. A larger sample size may therefore lead to larger and more numerous findings of CNS injury.

The sample size was limited because of the patient population selected. Severe pediatric HF is an uncommon disorder, and it is even less common to find cooperative, neurologically intact, and otherwise healthy children with isolated severe HF. In addition, the window of opportunity to perform nonsedated MRI on HF subjects is sometimes limited because of the progressive nature of the disease. Although a single-institution study provided ultimate control over MRI parameters and image acquisition, this structure is itself limiting to the sample size. Further studies will require a longer duration of recruitment or a multi-institutional study.

\section{Conclusion}

Pediatric HF subjects show regional loss of GM volume in specific areas of the brain essential for sympathetic and parasympathetic regulation, memory, mood, and perception of dyspnea and include the mid-hippocampus, the bilateral insular cortices, and the medial aspect of the superior frontal gyrus. Volume loss in these subjects occurs in the absence of thromboembolic disease and other comorbidities. The affected brain areas are similar to those injured in adult HF subjects and appear to be important in the pathophysiology of HF syndrome, which includes mood disturbances, impaired memory, and a propensity toward disease progression, arrhythmia, and sudden death.

Acknowledgments This work was supported by a grant from the University of Southern California's General Clinical Research Center, Los Angeles, CA, USA.

Open Access This article is distributed under the terms of the Creative Commons Attribution Noncommercial License which permits any noncommercial use, distribution, and reproduction in any medium, provided the original author(s) and source are credited.

\section{References}

1. Aggleton JP, Brown MW (1999) Episodic memory, amnesia, and the hippocampal-anterior thalamic axis. Behav Brain Sci 22: 425-444 
2. Almeida OP, Flicker L (2001) The mind of a failing heart: a systematic review of the association between congestive heart failure and cognitive functioning. Intern Med 31:290-295

3. Ancoli-Israel S, Duhamel ER, Stepnowski C, Engler R, CohenZion M, Marler M (2003) The relationship between congestive heart failure, sleep apnea, and mortality in older men. Chest 124:1400-1405

4. Arikuni T, Sako H, Murata A (1994) Ipsilateral connections of the anterior cingulate cortex with the frontal and medial temporal cortices in the macaque monkey. Neurosci Res 21:19-39

5. Ashburner J, Friston KJ (2000) Voxel-based morphometry-the methods. Neuroimage 11:805-821

6. Auslender M (2000) Pathophysiology of pediatric heart failure. Prog Pediatr Cardiol 11:175-184

7. Banzett RB, Mulnier HE, Murphy K, Rosen SD, Wise RJ, Adams L (2000) Breathlessness in humans activates insular cortex. Neuroreport 11:2117-2120

8. Critchley HD, Mathias CJ, Josephs O, O'Doherty J, Zanini S, Dewar BK et al (2003) Human cingulate cortex and autonomic control: converging neuroimaging and clinical evidence. Brain 126:2139-2152

9. Cross RL, Kumar R, Macey PM, Doering LV, Alger JR, Yan-Go FL et al (2008) Neural alterations and depressive symptoms in obstructive sleep apnea patients. Sleep 31:1103-1109

10. Cummings JL (1993) Frontal-subcortical circuits and human behavior. Arch Neurol 50:873-880

11. Data Format Working Group (2005) Available at: NIfTI.NIMH.NIH.gov/background. Accessed 30 Oct 2009

12. Demaso DR, Kelley SD, Bastardi H, O'Brien P, Blume ED (2004) The longitudinal impact of psychological functioning, medical severity, and family functioning in pediatric heart transplantation. J Heart Lung Transplant 23:473-480

13. Drevets WC (2000) Neuroimaging studies of mood disorders. Biol Psychiatry 48:813-829

14. du Boisgueheneuc F, Levy R, Volle E, Seassau M, Duffau H, Kinkingnehun $\mathrm{S}$ et al (2006) Functions of the let superior frontal gyrus in humans: a lesion study. Brain 129:3315-3328

15. Esler M, Kaye D, Lambert G, Esler D, Jennings G (1997) Adrenergic nervous system in heart failure. Am $\mathrm{J}$ Cardiol 80(11A):7L-14L

16. Fernandez G, Weyerts H, Schrader-Bolsche M, Tendolkar I, Smid HG, Templemann C et al (1998) Successful verbal encoding into episodic memory engages the posterior hippocampus: a parametrically analyzed functional magnetic resonance imaging study. J Neurosci 18:1841-1847

17. Gianaros PJ, Derbyshire SWG, May JC, Siegle GJ, Gamalo MA, Jennings JR (2005) Anterior cingulate activity correlates with blood pressure during stress. Psychophysiology 42:627-635

18. Glausser TA, Rorke LB, Weinberg PM, Clancy RR (1990) Acquired neuropathologic lesions associated with the hypoplastic left heart syndrome. Pediatrics 85:991-1000

19. Good CD, Johnsrude IS, Ashburner J, Henson RNA, Friston KJ, Frackowiak RSJ (2001) A voxel-based morphometry study of ageing in 465 normal adult human brains. Neuroimage 14:21-36

20. Guzzetti S, Cogliati C, Turiel M, Crema C, Lombardi F, Malliani A (1995) Sympathetic predominance followed by functional denervation in the progression of chronic heart failure. Eur Heart J 16:1100-1107

21. Harper RM, Bandler R, Spriggs D, Alger JR (2000) Lateralized and widespread brain activation during transient blood pressure elevation revealed by magnetic resonance imaging. J Comp Neurol 417:195-204

22. Harper RM, Macey PM, Henderson LA, Woo MA, Macey KE, Frysinger RC et al (2003) fMRI responses to cold pressor challenges in control and obstructive sleep apnea subjects. J Appl Physiol 94:1583-1595
23. Havranek EP, Ware MG, Lowes BD (1999) Prevalence of depression in congestive heart failure. Am J Cardiol 84:348-350

24. Henderson LA, Woo MA, Macey PM, Macey KE, Frysinger RC, Alger JR et al (2003) Neural responses during Valsalva maneuvers in obstructive sleep apnea syndrome. J Appl Physiol 94:1063-1074

25. Jankowska EA, Ponikowski P, Piepoli MF, Benasiak W, Anker SD, Poole-Wilson PA (2006) Autonomic imbalance and immune activation in chronic heart failurēpathophysiological links. Cardiovasc Res 70:434-445

26. Jiang W, Kuchibhatla M, Cuffe MS, Christopher EJ, Alexander JD, Clary GL et al (2004) Prognostic value of anxiety and depression in patients with chronic heart failure. Circulation 110:3452-3456

27. Kumar R, Woo MA, Birrer BV, Macey PM, Fonarow GC, Hamilton MA et al (2009) Mammillary bodies and fornix fibers are injured in heart failure. Neurobiol Dis 33:236-242

28. Laakso MP, Vaurio O, Koivisto E, Savolainen L, Eronen M, Aronen HJ et al (2001) Psychopathy and the posterior hippocampus. Behav Brain Res 118:187-193

29. Mahle WT, Wernovsky G (2001) Long-term developmental outcome of children with complex congenital heart disease. Clin Perinatol 28:235-247

30. Mahle WT, Tavani F, Zimmerman RA, Nicholson SC, Galli KK, Gaynor JW et al (2002) An MRI study of neurological injury before and after congenital heart surgery. Circulation 106:I109I114

31. Majnemer A, Limperopoulos C (1999) Developmental progress of children with congenital heart defects requiring open heart surgery. Semin Pediatr Neurol 6:12-19

32. Massin M, von Bernuth G (1998) Clinical and haemodynamic correlates of heart rate variability in children with congenital heart disease. Eur J Pediatr 157:967-971

33. Menteer JD, Woo MS, So JD, Lewis AB (2007) Symptoms of dysautonomia, sleep disturbance, and abnormal cognition in pediatric heart failure. Pediatr Cardiol 28:379-384

34. Murberg TA, Bru E, Svebak S, Tveteras R, Aarsland T (1999) Depressed mood and subjective health symptoms as predictors of mortality in patients with congestive heart failure: a two-year follow-up study. Int J Psychiatry Med 29:311-326

35. Ohuchi H, Takasugi H, Ohashi H, Okada Y, Yamada O, Ono Y et al (2003) Stratification of pediatric heart failure on the basis of neurohormonal and cardiac autonomic nervous activities in patients with congenital heart disease. Circulation 108:23682376

36. Opie LH (2002) The neuroendocrinology of congestive heart failure. Cardiovasc J S Afr 13:171-178

37. Oppenheimer S (2006) Cerebrogenic cardiac arrhythmias: cortical lateralization and clinical significance. Clin Auton Res 16:611

38. Oppenheimer SM, Wilson JX, Guiraudon C, Cechetto DF (1991) Insular cortex stimulation produces lethal cardiac arrhythmias: a mechanism of sudden death? Brain Res 550:115-121

39. Oppenheimer SM, Gelb A, Girvin JP, Hachinski VC (1992) Cardiovascular effects of human insular cortex stimulation. Neurology 42:1727-1732

40. Oppenheimer SM, Kedem G, Martin WM (1996) Left-insular cortex lesions perturb cardiac autonomic tone in humans. Clin Auton Res 6:131-140

41. Packer M (1992) The neurohormonal hypothesis: a theory to explain the mechanism of disease progression in heart failure. $\mathrm{J}$ Am Coll Cardiol 20:248-254

42. Packer M, Bristow MR, Cohn JN, Colucci WS, Fowler MB, Gilbert EM et al (1996) The effect of carvedilol on morbidity and mortality in patients with chronic heart failure. New Engl J Med 334(21):1349-1355 
43. Pfeiffer C, Proline JB, Thivard L, Aubier M, Samson Y (2001) Neural substrates for the perception of acutely induced dyspnea. Am J Respir Crit Care Med 163:951-957

44. Putzke JD, Williams MA, Rayburn BK, Kirklin JK, Boll TJ (1998) The relationship between cardiac function and neuropsychological status among heart transplant candidates. J Card Fail 4:295-303

45. Roman DD, Kubo SH, Ormaza S, Francis GS, Blank AJ, Shumway SJ (1997) Memory improvement following cardiac transplantation. J Clin Exp Neuropsychol 19:692-697

46. Schmidt R, Fazekas F, Offenbacher H, Dusleag J, Lechner H (1991) Brain magnetic resonance imaging and neuropsychologic evaluation of patients with idiopathic dilated cardiomyopathy. Stroke 22:195-199

47. Spurkland I, Bjorbaek T, Hagemo P (2001) Psychosocial functioning in children after transplantation of the heart, and heart and lungs. Cardiol Young 11:277-284

48. Tang WH, Francis GS (2005) Neurohormonal upregulation in heart failure. Heart Fail Clin 1:1-9

49. Todaro JF, Fennell EB, Sears SF, Rodrigue JR, Roche AK (2000) Review: cognitive and psychological outcomes in pediatric heart transplantation. J Pediatr Psychol 25:567-576

50. Uzark K, Lincoln A, Lamberti JJ, Mainwaring RD, Spicer RL, Moore JW (1997) Neurodevelopmental outcomes in children with Fontan repair of functional single ventricle. Pediatrics 101:630-633
51. Vaccarino V, Kasl SV, Abramson J, Krumholz HM (2001) Depressive symptoms and risk of functional decline in patients with heart failure. J Am Coll Cardiol 38:199-205

52. Watanabe K, Matsui M, Matsuzawa J, Tanaka C, Noguchi K, Yoshimura $\mathrm{N}$ et al (2009) Impaired neuroanatomic development in infants with congenital heart disease. J Thorac Cardiothorac Surg 137:146-153

53. Wernovsky G, Stiles KM, Gauvreau K, Gentles TL, du Plessis AJ, Bellinger DC et al (2000) Cognitive development after the Fontan operation. Circulation 102:883-889

54. Wernovsky G, Shillingford AJ, Gaynor JW (2005) Central nervous system outcomes in children with complex congenital heart disease. Curr Opin Cardiol 20:94-99

55. Woo MA, Macey PM, Fonarrow GC, Hamilton MS, Harper RM (2003) Regional brain gray matter loss in heart failure. J Appl Physiol 95:677-684

56. Woo MA, Macey PM, Keens PT, Kumar R, Fonarrow GC, Hamilton MA et al (2005) Functional abnormalities in brain areas that mediate autonomic nervous system control in advanced heart failure. J Cardiac Fail 11:437-446

57. Wray J, Radley-Smith R (2006) Longitudinal assessment of psychological functioning in children after heart or heart-lung transplantation. J Heart Lung Transpl 25:345-352

58. Zhang ZH, Rashba S, Oppenheimer SM (1998) Insular cortex lesions alter baroreceptor sensitivity in the urethane-anesthetized rat. Brain Res 813:73-81 